\title{
Sciendo
}

Transport and Telecommunication, 2022, volume 23, no. 1, 25-32

Transport and Telecommunication Institute, Lomonosova 1, Riga, LV-1019, Latvia

DOI 10.2478/ttj-2022-0003

\section{A KALMAN FILTER BASED HYBRID ROUTING PROTOCOL FOR EFFICIENT VEHICLE CONNECTIVITY AND TRAFFIC MANAGEMENT}

\author{
Divya Punia ${ }^{1}$, Rajender Kumar ${ }^{2}$ \\ ${ }^{I}$ National Institute of Technology Kurukshetra, Haryana, India \\ dpunia92@gmail.com \\ ${ }^{2}$ National Institute of Technology Kurukshetra, Haryana, India \\ rkumar.kkr@gmail.com
}

The geographic routing protocols in Vehicular Ad Hoc Networks (VANETs) are contemplated as most efficacious protocols. Though, such types of protocols communicate a huge quantity of data that influence the network connectivity negatively. Also, out of bound issue is the second major disadvantage of geographic routing protocols. To provide a solution to these impediments, a novel K-PGRP (Kalman filter-Predictive Geographic Routing Protocol) routing protocol is proposed in this paper. K-PGRP is an improvement to PGRP (Predictive Geographic Routing Protocol) routing protocol and wields Kalman filter as a prediction module in PGRP routing protocol in order to anticipate the neighbor location and to select the propitious neighbor for advancing packets in both urban and highway framework which leads to efficient connectivity in the network and improves road safety. K-PGRP is then compared with PGRP, GPSR (Greedy Perimeter Stateless Routing) and GPCR (Greedy Perimeter Coordinator Routing) routing protocols in terms of throughput and packet delivery ratio metrics and outperformed all the simulation cases. The simulations were performed on MATLAB R2018a along with traffic simulator SUMO.

Keywords: Routing, Kalman filter, IoV, prediction, connectivity

\section{Introduction}

VANET is a propitious innovation for developing ITS (Intelligent Transportation System). Yet, routing in VANETs is a strenuous job because of environmental circumstances and dynamic system topology. Nowadays, all over the world, millions recently enrolled vehicles are outfitted with GPS receivers and route frameworks. Accordingly, geographic routing protocols (Boussoufa-Lahlah et al., 2018) are well known in VANETs as a result of their effortlessness, accessibility of GPS gadgets and low overhead. Technical and environmental conditions affect GPS accuracy, leading to deviation in vehicle actual and GPS predicted position by a marginal error of \pm 2-100 $\mathrm{m}$. In VANETs, the connectivity amid two nodes and the system configuration is legitimate just for a brief time frame due to the diverse speed and high mobility of nodes. Conversely, in VANETs most geographic routing protocols consider that node speed is static in time period of hello packet and that each vehicle distinguishes its neighbors utilizing a hello packet. Exemplary routing protocols which reinforce static node mobility are GPCR (Greedy Perimeter Coordinator Routing) (Lochert et al., 2003), GPSR (Greedy Perimeter Stateless Routing) (Karp and Kung, 2003), CAR (Connectivity-Aware Routing) (Naumov and Gross, 2007) etc. Moreover, when vehicles over-take/ pass each other, they experience diverse acceleration rates which results in increasing end-to-end delay and number of hops and this is one more issue faced by geographic routing protocols. Therefore, anticipating neighbor location and forwarding strategies for every vehicle can be wielded to upgrade the network connectivity both in a highly mobile ambience like highway scenario and low to medium velocity ambience like urban scenario (Bao et al., 2020; Cheng et al., 2009; Artimy et al., 2005). PGRP (Karimi and Shokrollahi, 2018) as a recent georouting protocol wields predictive greedy forwarding approach in order to anticipate near future location of neighboring vehicles and encounter many constraints. Firstly, PGRP wields local information by discerning one-hop neighbors. Therefore, every vehicle advances the packet which is accurate from local point of view, i.e., follows greedy approach and this conventional greedy concept is applied on the future predicted positions. Thus, at every instant, PGRP fails to provide a global optimum solution as it discerns one- hop neighbors based on local information only. Secondly, in order to anticipate neighbor vehicle location, the present node (i.e., vehicle) in PGRP assign weight to neighboring vehicles based on direction and distance parameters only and velocity parameter is not considered which can degrade the connectivity of two vehicles in high mobility environment leading to high packet loss and degrading throughput. Thirdly, PGRP is applied in 
both urban and highway environment and instead of applying a real urban environment, it incorporates a simple grid layout for urban environment. Although, PGRP predicts neighbor location but lacks in precise location prediction of neighboring vehicle and also QoS parameters degrades in high mobility real environment. Thus, to upgrade the connectivity in georouting protocols and to overcome the shortcomings of PGRP protocol and state of the art geographic routing protocols mentioned above, this paper presents a novel K-PGRP (Kalman filter- Predictive Geographic Routing Protocol) routing protocol for IoV (Internet of Vehicle) environment. Categorically, this paper provides following contributions: 1) Implements PGRP protocol and integrate and adapts the Kalman filter in PGRP algorithm to predict location of neighbor precisely and resolves Out of bound issue in VANETs; 2) Demonstrates the effect of vehicle density on neighbor location prediction through MATLAB simulations; 3) Quantitative comparison and performance estimation with the existing state of the art protocols in real-world highway and urban vehicular environment. To the best of our knowledge, no effort has been made to resolve routing issues for the IoV network, all the research has been carried out on VANETs only.

\section{K-PGRP: A hybrid routing approach employing kalman filter and PGPR routing technique}

The paper suggests a novel K-PGRP routing protocol which wields Kalman filter as a prediction module in PGRP routing technique in order to anticipate the neighbor location and to select the propitious neighbor for advancing packets in urban and highway framework. Therefore, a beacon packet format in K-PGRP encloses velocity of nodes $\left(v_{X}, v_{Y}\right)$ along latitude and longitude, acceleration of nodes $\left(a_{X}, a_{Y}\right)$ along with vehicle_Id, and location of the nodes $\left(l_{X}, l_{Y}\right)$. Direction and speed are transmitted as velocity vectors. The $\mathrm{l}_{\mathrm{X}}$ and $\mathrm{l}_{\mathrm{Y}}$ coordinate i.e., location of vehicle in $\mathrm{x}$ and $\mathrm{y}$ coordinates are illustrated by following equations (1) and (2).

$\mathrm{l}_{\mathrm{X}(\mathrm{t})}=\mathrm{l}_{\mathrm{X}(\mathrm{t}-1)}+\mathrm{v}_{\mathrm{X}(\mathrm{t}-1)} \times \mathrm{dt}+\frac{\mathrm{a}_{\mathrm{X}(\mathrm{t}-1)} \times \mathrm{dt}^{2}}{2}$.

$\mathrm{l}_{\mathrm{Y}(\mathrm{t})}=\mathrm{l}_{\mathrm{Y}(\mathrm{t}-1)}+\mathrm{v}_{\mathrm{Y}(\mathrm{t}-1)} \times \mathrm{dt}+\frac{\mathrm{a}_{\mathrm{Y}(\mathrm{t}-1)} \times \mathrm{dt}^{2}}{2}$.

Where: $\mathrm{l}_{\mathrm{X}(\mathrm{t}-1)}, \mathrm{l}_{\mathrm{Y}(\mathrm{t}-1)}, \mathrm{v}_{\mathrm{X}(\mathrm{t}-1)}, \mathrm{v}_{\mathrm{Y}(\mathrm{t}-1)}, \mathrm{a}_{\mathrm{X}(\mathrm{t}-1)}$ and $\mathrm{a}_{\mathrm{Y}(\mathrm{t}-1)}$ are location, velocity and acceleration of node at time stamp $t$ and $\mathrm{dt}$ is the difference of time in change of velocity. Also, the format of every node in neighbor record includes vehicle $I d,\left(l_{X}, l_{Y}\right)$ and $\left(v_{X}, v_{Y}\right)$ of neighbors and sextuple $S_{t}$ (Kalman state vector), which is described as $\left[\overline{1}_{X}, v_{X}, a_{X}, l_{Y}, v_{Y}, a_{Y}\right]$. K-PGRP routing algorithm employs a 3-step procedure as follows:

\section{To design and initialize the records of neighbor and system state $\left(\mathrm{X}_{\mathrm{K}}\right)$}

Initially, K-PGRP approach exchanges the system state $\left(\mathrm{S}_{\mathrm{t}}\right)$ details. The vector $\mathrm{S}_{\mathrm{t}}$ represents the system state at time stamp $(\mathrm{t})$, which illustrates the least data set required to elucidate the system dynamic functioning. It is assumed that the current state (at time stamp $\mathrm{t}$ ) evolves from previous state ( $\mathrm{t}-1)$.

The periodic beacon signal is wielded by every node to inform its proximate neighboring node about its current velocity and location at current time. After receiving beacon packet, every node update new neighbor entry in its neighboring record and initialize sextuple $S_{t}$ state vector which by following equation:

$\mathrm{S}_{\mathrm{t}}=\mathrm{MS}_{\mathrm{t}-1}+\mathrm{N}_{\mathrm{t}}$.

Where M: State transition matrix and $\mathrm{N}_{\mathrm{t}}$ : System error noise at time stamp $\mathrm{t}$. Also, the measurement vector $Y_{t}$ appends the GPS received measurement to the $S_{t}$ by following equation:

$\mathrm{Y}_{\mathrm{t}}=\mathrm{HS}_{\mathrm{t}}+\mathrm{Z}_{\mathrm{t}}$.

Where H: Measurement model matrix which sustains a relationship amid system states and measured vector and $Z_{t}$ : Measurement noise on GPS at time stamp $t$.

\section{To monitor the mobility of neighbors and checking for out of bound}

In a real-world IoV environment, due to high mobility of vehicles, out of bound issue (Karimi and Shokrollahi, 2018) takes place i.e., the previously received neighbors' information may have altered before receiving the next beacon packet. Figure 1 illustrates out of bound issue in which, vehicle $\mathrm{X}$ is a source vehicle and has neighboring vehicles $\mathrm{Y}$ and $\mathrm{Z}$ in its transmission range. $\mathrm{X}$ wants to forward hello packet to moving destination vehicle D. All vehicles are moving at different high speed. At time t, Y and 
$\mathrm{Z}$ are having the hello packet and $\mathrm{Z}$ is nearest to $\mathrm{D}$. At time $\mathrm{t}_{1}$, the packet is forwarded to $\mathrm{Z}$ but $\mathrm{Z}$ goes out of bound of $X$ i.e., not in the transmission range of $X$ due to high speed and the packet will be dropped which leads to the out of bound problem.

Thus, out of bound issue is resolved wielding one-step-advanced estimation equation as follows:

$S_{t \mid t-1}=\mathrm{MS}_{\mathrm{t}-1}+\mathrm{N}_{\mathrm{t}}$

Where $S_{t \mid t-1}$ : Priori state vector which is estimated by $\mathrm{S}_{\mathrm{t}-1}$ (previous state vector).

The equation (5) recursively anticipates new neighbor state $S_{t \mid t-1}$ at every time stamp t. Then, equation (6) estimates the error covariance as follows:

$\mathrm{E}_{\mathrm{t} \mid \mathrm{t}-1}=\mathrm{ME}_{\mathrm{t}-1} \mathrm{M}^{\mathrm{T}}+\mathrm{Q}_{\mathrm{t}-1}$

Where $\mathrm{E}_{\mathrm{t} \mid \mathrm{t}-1}$ : Covariance matrix of the priori error, Q: Process noise covariance and $\mathrm{M}^{\mathrm{T}}$ : Transpose of vector M. By equation (5) and (6), every node in real time can reconstruct and follow its neighboring node state.

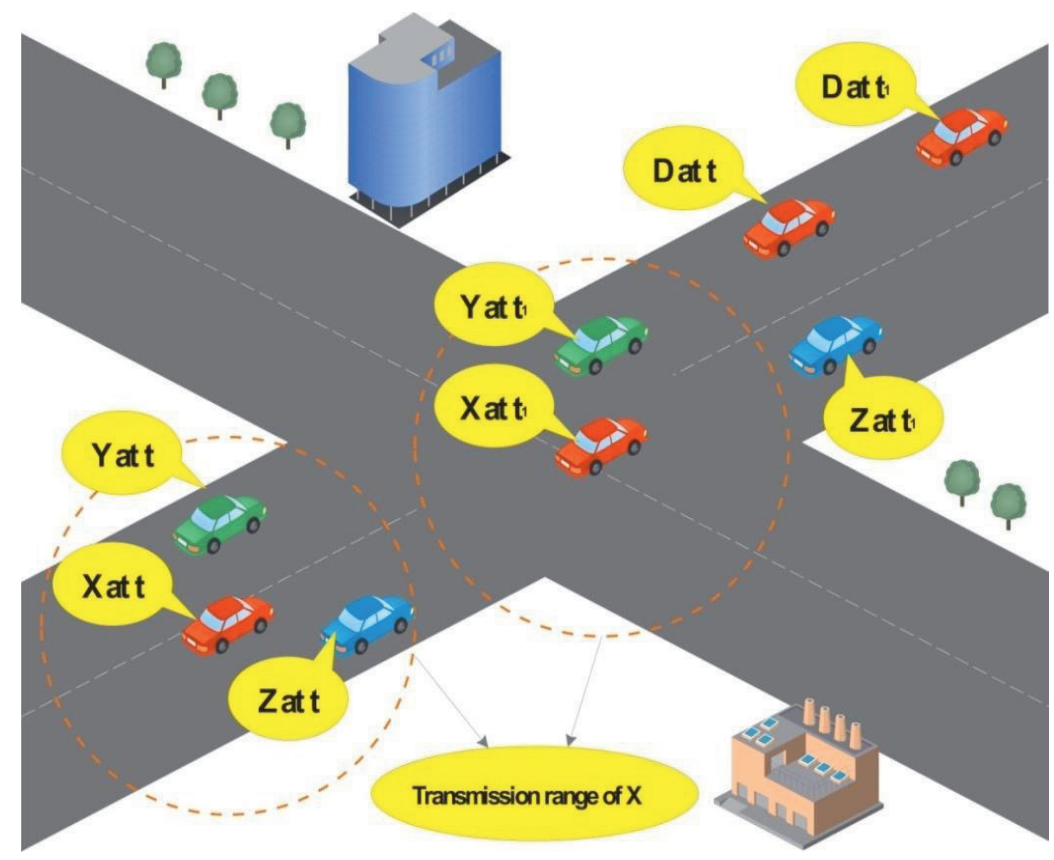

Figure 1. Out of bound issue in VANET

\section{To observe, correct and update the estimates}

On the basis of observation and estimation of $\mathrm{S}_{\mathrm{t}}$, the $\mathrm{KF}$ optimizes the correction weight in order to provide precise estimations. The correction i.e., difference amid predicted and actual observation is given by below equation:

$\mathrm{D}=\mathrm{Y}_{\mathrm{t}}-\mathrm{HS}_{\mathrm{t} \mid \mathrm{t}-1}$

Where D: Kalman change. Subsequently, kalman gain $\left(\mathrm{K}_{\mathrm{t}}\right)$ is calculated by following equation:

$\mathrm{K}_{\mathrm{t}}=\mathrm{E}_{\mathrm{t} \mid \mathrm{t}-1} \mathrm{H}^{\mathrm{T}}\left(\mathrm{HE}_{\mathrm{t} \mid \mathrm{t}-1} \mathrm{H}^{\mathrm{T}}+\mathrm{R}\right)^{-1}$.

Where R: Measurement noise covariance. After evaluating $\mathrm{K}_{\mathrm{t}}$, the updated state estimate $\left(\mathrm{S}_{\mathrm{t} \mid \mathrm{t}}\right)$ and error covariance $\left(\mathrm{E}_{\mathrm{t} \mid \mathrm{t}}\right)$ are calculated by equation (9) and (10) respectively.

$\mathrm{S}_{\mathrm{t} \mid \mathrm{t}}=\mathrm{S}_{\mathrm{t} \mid \mathrm{t}-1}+\mathrm{K}_{\mathrm{t}} \mathrm{D}$.

$\mathrm{E}_{\mathrm{t} \mid \mathrm{t}}=\left(1-\mathrm{S}_{\mathrm{t}} \mathrm{H}\right) \mathrm{E}_{\mathrm{t} \mid \mathrm{t}-1}$.

Thus, $K_{t}$ is wielded to weight the altered values and provide the information which is appended to update state estimate. The algorithm for location prediction of vehicles is illustrated in Figure 2. 


\section{K-PGRP Algorithm for location prediction}

Input data: Source node receive information from neighboring node via beacon packet

Result: Location prediction of nodes

Source node broadcast HELLO packet in network

Neighbor node transmit beacon packet to source node

for every $i=1$ to $n$ do $\quad n$ : Number of neighboring nodes

get location, velocity and distance measurement via KF

for each node do

initialize $\mathrm{S}_{\mathrm{t}}$ Vector

repeat for each time epoch $\mathrm{t}$

estimate $S_{t \mid t-1}$ and $E_{t \mid t-1}$

check for out of bound and return new value of estimate $S_{t \mid t-1}$

update neighbour list by incorporating new output from $S_{t \mid t-1}$

until $\mathrm{T} \neq \mathrm{T}_{\text {threshold }}$

end

while $\mathrm{T} \neq \mathrm{T}_{\text {threshold }}$ and beacon packets are received from neighbors do calculate change $(\mathrm{D})$ and $\left(\mathrm{K}_{\mathrm{t}}\right)$

update $S_{\mathrm{t} \mid \mathrm{t}}$ and $\mathrm{E}_{\mathrm{t} \mid \mathrm{t}}$

update neighbour list by incorporating new output from $S_{t \mid t}$

return $S_{t \mid t}$

end while

end

Figure 2. Algorithm for next node selection using K-PGRP

\section{Simulation Results}

The experiments were carried out on MATLAB R2018a along with traffic simulator SUMO (Simulation of Urban Mobility) in order to evaluate the performance of the proposed routing algorithm (K-PGRP). TraCI4Matlab (Acosta and Espinosa, 2005) was wielded in order to enable client-server mechanism amid Matlab (client) and SUMO (server). SUMO was wielded to create real world urban scenario of Bengaluru city, India. Bengaluru is considered for simulation because according to report of location technology company TomTom for 2020 year, Bengaluru takes the title of highly congested city in the world (TOMTOM, 2020). Also, Texas State Highway 130 is considered for highway scenario generation, as it is one of the high-speed highways in the world. Therefore, both the scenarios meet prerequisites for K-PGRP algorithm. Simulation settings for K-PGRP are described in Table 1.

Table 1. General framework specifications

\begin{tabular}{|l|l|}
\hline Parameter & Specification \\
\hline Number of vehicles & $30,60,90,120,150,180,210,240,270,300$ \\
\hline Transmission range & $350 \mathrm{~m}$ \\
\hline Simulation area & $\begin{array}{l}\text { Highway }(10,000) \mathrm{m} \\
\text { Urban }(3500 \times 3500) \mathrm{m}\end{array}$ \\
\hline Propagation Model & Two-ray ground \\
\hline Maximum vehicle speed & $31.068 \mathrm{mph}-62.137 \mathrm{mph}$ \\
\hline Packet Length & 512 bytes \\
\hline
\end{tabular}




\begin{tabular}{|l|l|}
\hline Parameter & Specification \\
\hline Length of vehicle & $5 \mathrm{~m}$ \\
\hline Beacon interval & $1 \mathrm{~s}$ \\
\hline Simulation time & $500 \mathrm{~s}$ \\
\hline Vehicle acceleration & $2.4 \mathrm{~m} / \mathrm{s}^{2}$ \\
\hline Type of antenna & Omni-directional \\
\hline Vehicle deceleration & $4.5 \mathrm{~m} / \mathrm{s}^{2}$ \\
\hline PHY-MAC protocol & IEEE $802.11 \mathrm{p}$ \\
\hline
\end{tabular}

The effectiveness of the proposed K-PGRP routing is analyzed in urban and highway scenario by varying vehicle density and critically examined by contrasting the results with PGRP, GPCR and GPSR geographic routing protocols in terms of QoS metrics namely PDR and throughput as illustrated below.

\subsection{Packet Delivery Ratio (PDR)}

It is described as the total successful packets delivered to the destination node by total packets transmitted from source node. Figure 3 (a) and 3(b) describe PDR of K-PGRP, PGRP, GPSR and GPCR routing protocols in urban and highway scenario respectively. K-PGRP illustrates remarkable improvement in performance over PGRP, GPCR and GPSR protocol of 16.55\%, 38.75\% and 35.85\% respectively in urban scenario. Also, in highway scenario K-PGRP outperformed PGRP, GPCR and GPSR protocol by $13.99 \%, 39.16 \%$ and $31.86 \%$ respectively, but results demonstrate that in highway due to sparse network connection and high mobility of vehicles, PDR obtained is less w.r.t urban scenario in all the cases.

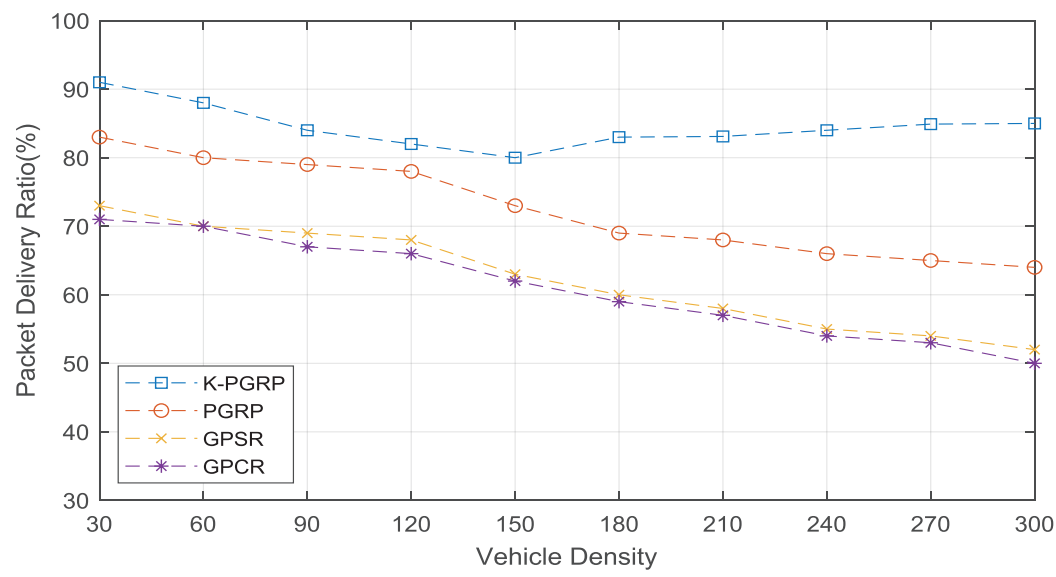

3(a) PDR (Urban Scenario)

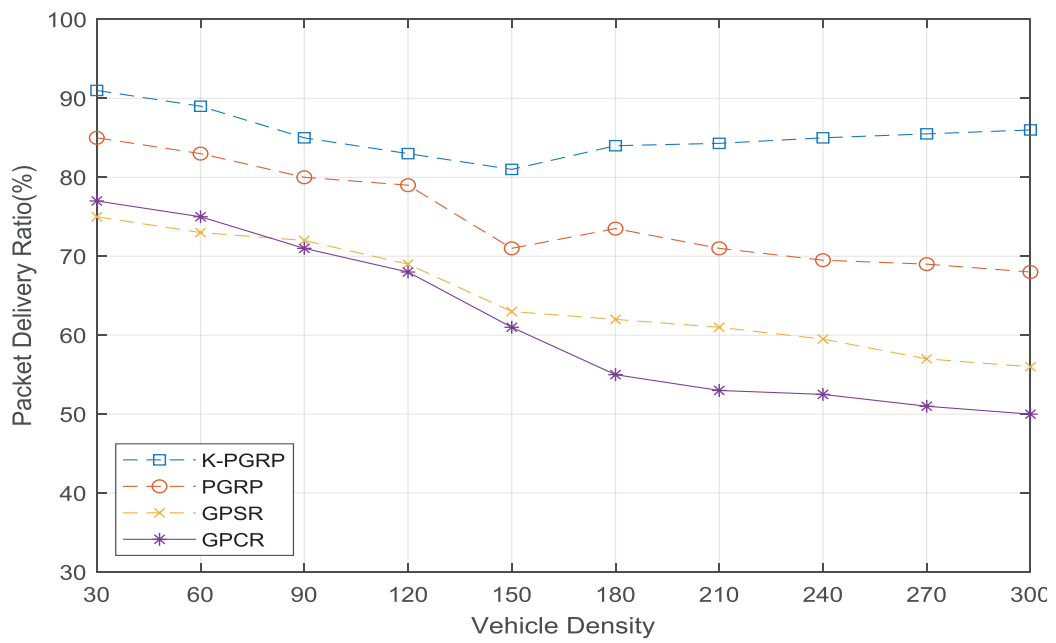

3(b) PDR (Highway Scenario)

Figure 3. The effect of node density on the behavior K-PGRP, PGRP, GPSR and GPCR protocols in terms of their PDR is depicted in (a) urban scenario and (b) highway scenario 
The GPCR and GPSR showed very low PDR in both the scenarios due to network disconnection and lack of node prediction strategy. Whereas, K-PGRP and PGRP demonstrate significant performance as these can predict the available neighbor hop for packet transmitted. Moreover, the channel contention increases as the vehicular density exceed 150 in both the scenarios which dwindles the performance of PGRP but K-PGRP still perform better due to the employed KF robustness which eradicate the issue of not having adequate neighbors to transmit packet. The K-PGRP performance was evaluated by the following [8] equation (11).

$\left(\frac{\text { The average aggregate of PDR in K-PGRP }}{\text { The average aggregate of PDR in other protocols }}-1\right) \times 100$.

\subsection{Throughput}

It is the amount of successfully transmitted information per unit time over a communication channel. It is measured in Kbps (Kilobits per second). Figure 4(a) and 4(b) describe throughput of KPGRP, PGRP, GPSR and GPCR routing protocols in urban and highway scenario respectively. K-PGRP illustrates remarkable improvement in performance over PGRP, GPCR and GPSR protocol of $14.88 \%$, $39.52 \%$ and $35.14 \%$ respectively in urban scenario. Also, in highway scenario K-PGRP outperformed PGRP, GPCR and GPSR protocol by $13.60 \%, 38.75 \%$ and $34.83 \%$ respectively, but results demonstrate that in highway due to sparse network, throughput obtained is less w.r.t urban scenario in all the cases.

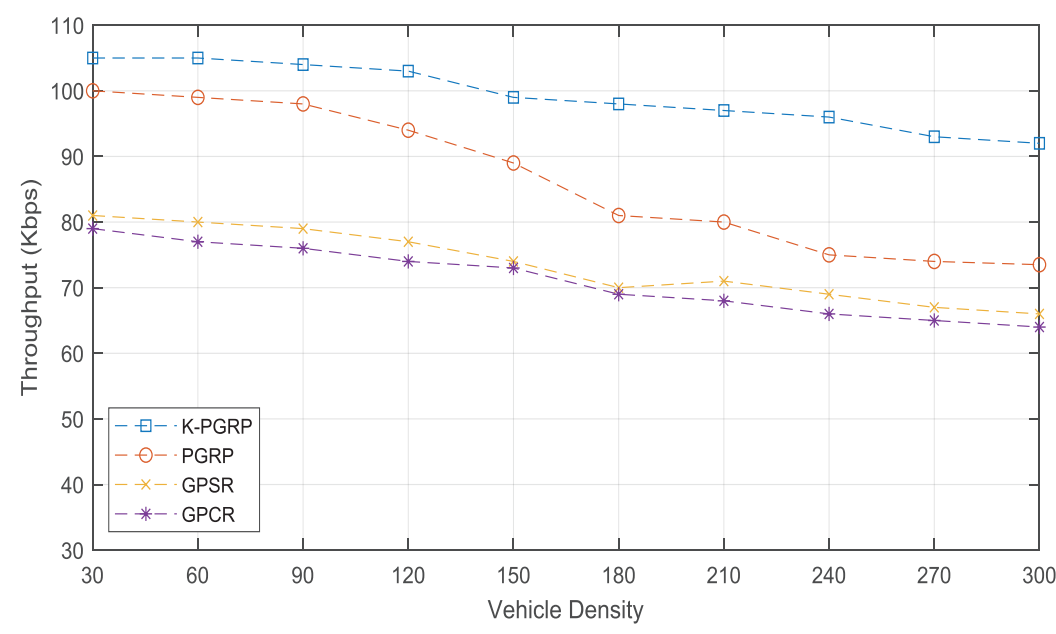

4(a) Throughput (Urban Scenario)

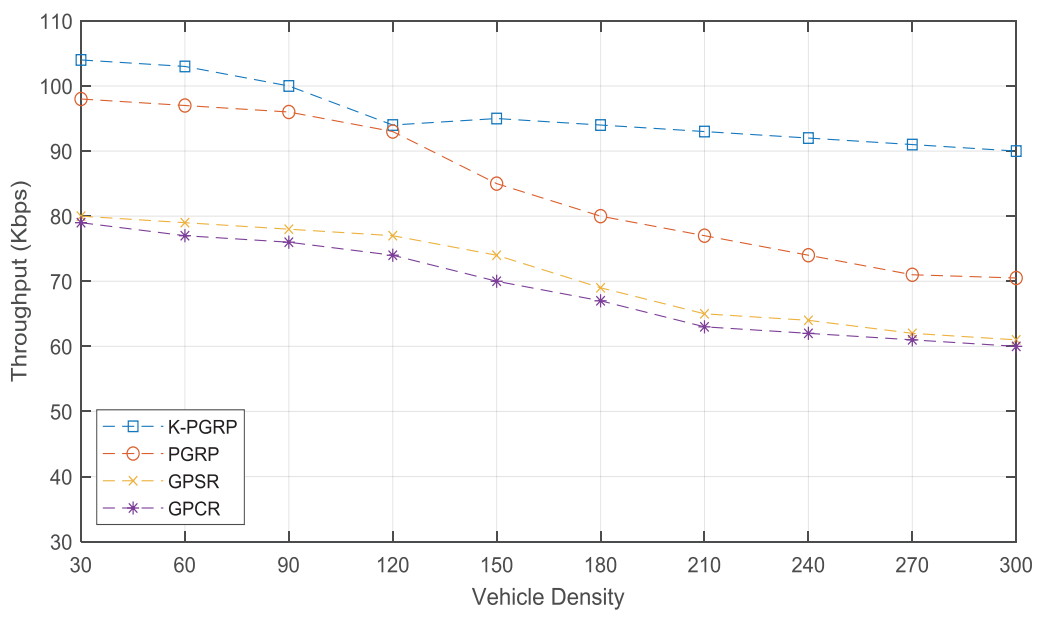

4(b) Throughput (Highway Scenario)

Figure 4. The effect of node density on the behavior K-PGRP, PGRP, GPSR and GPCR protocols in terms of their throughput is depicted in (a) urban scenario and (b) highway scenario 
When the density of vehicles exceeds 150 , wielding PGRP alone decreases the throughput because packets are steered to congested region of the network. Therefore, employing KF in PGRP will steer the packet to less congested regions which results in increased throughput. The K-PGRP performance was evaluated by the following equation (12).

$\left(\frac{\text { The average aggregate of throughput in K-PGRP }}{\text { The average aggregate of throughput in other protocols }}-1\right) \times 100$.

Figure 5(a) and 5(b) graphically illustrates the difference between the predicted location of KPGRP and PGRP protocol in both urban (Bengaluru) and highway scenario (Texas State Highway 130). GPCR and GPSR protocols lack this prediction strategy i.e., why they are not shown in the plot. The plots present the actual map of Bengaluru city and Texas state highway on which vehicles/nodes traverse.

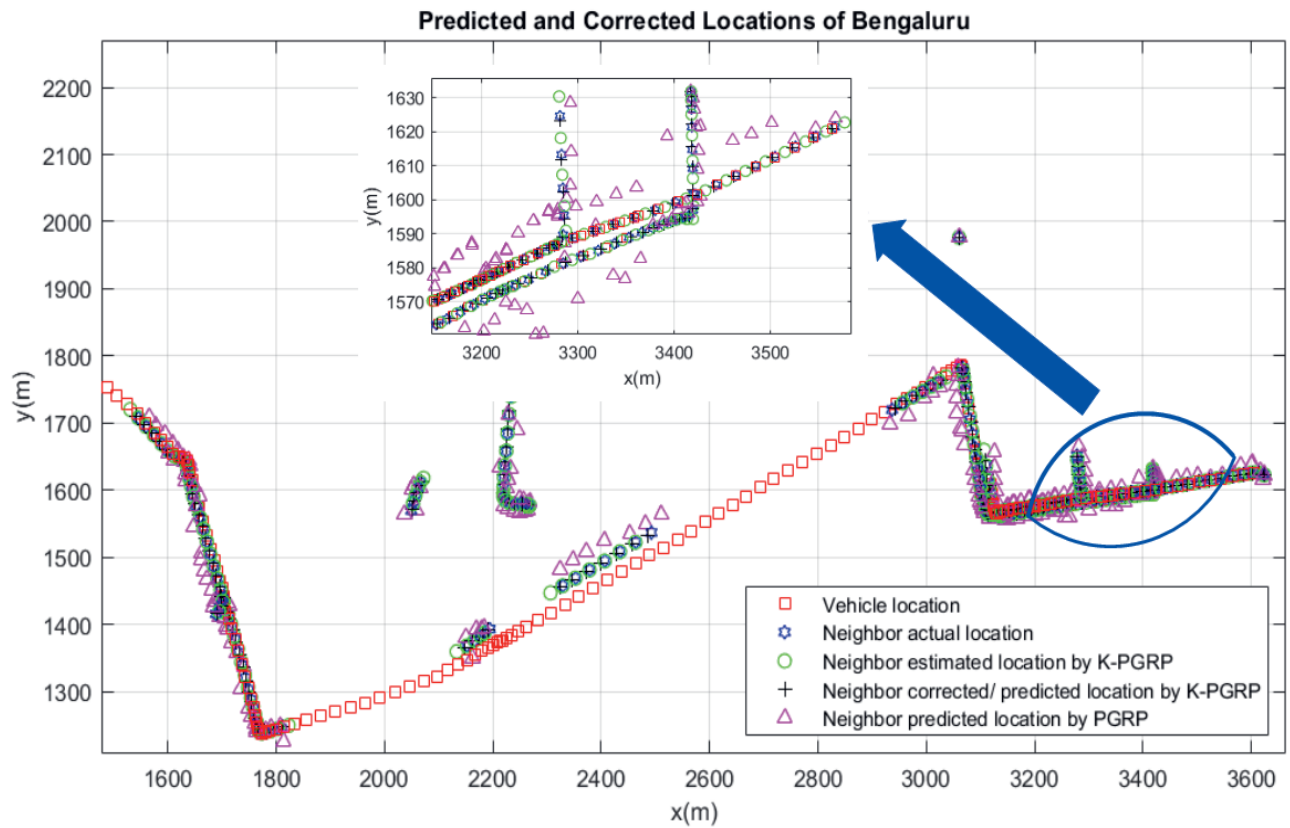

5(a) Bengaluru Map (Urban Scenario)

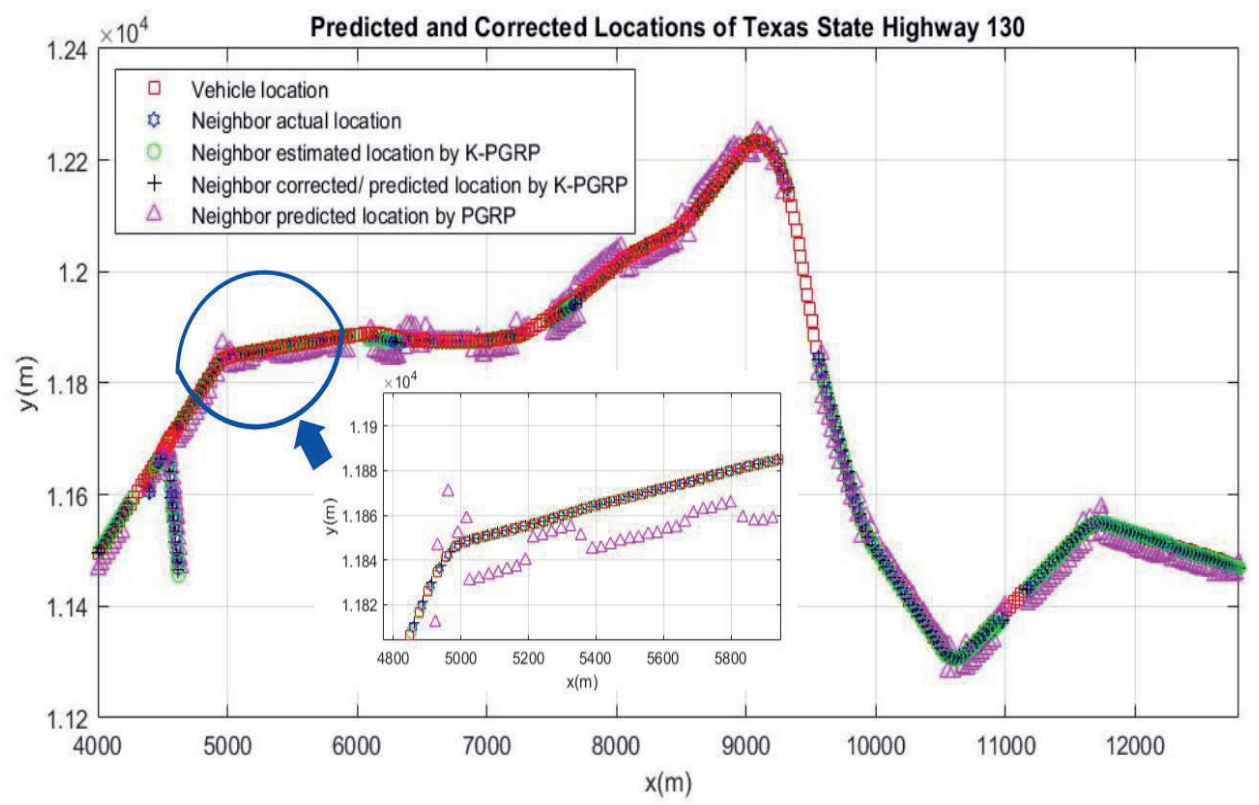

5(b) Texas State Highway 130 Map (Highway Scenario)

Figure 5. The effect of K-PGRP and PGRP routing protocol to predict neighbor location in (a) urban scenario and (b) highway scenario 
Figure 5 depicts the actual neighbor location, K-PGRP estimated location of the neighbor and the location of neighbor estimated by PGRP. A magnified plot of small area is also embedded in both the scenarios, in order to demonstrate the accurate location prediction by K-PGRP and PGRP protocol. In both figure 5(a) and 5(b), it is very clear that actual neighbor location and K-PGRP estimated location of the neighbor nearly overlap whereas location of neighbor estimated by PGRP is far away from actual neighbor location, showing that K-PGRP predicted the location of vehicles more accurately than PGRP protocol and thus improved the overall network connectivity.

\section{Conclusion and Future Scope}

To anticipate the neighbor location and to select the propitious neighbor for advancing packets is significant for the efficacy of geographic routing protocols in both urban and highway scenario. The paper presents a novel K-PGRP routing protocol which wields kalman filter in order to estimate the location of current neighbor, and it is an improvement to PGRP geographic routing protocol. The experimental simulations were performed using MATLAB R2018a and traffic simulator SUMO. The potential of K-PGRP routing protocol was analyzed by contrasting its simulation results with PGRP, GPSR and GPCR routing protocols in terms of throughput and PDR metrics. The simulation results present that K-PGRP improved overall PDR up to a minimum of $13.99 \%$, and also improved throughput up to a minimum average of $13.60 \%$ at best w.r.t PGRP protocol. Certainly, the accuracy is ameliorated wielding K-PGRP routing protocol irrespective of traffic scenarios (highway/urban) and eventually provides road safety. Therefore, the proposed protocol can be contemplated for anticipation intend and optimization in IoV. In future, we plan to implement extended kalman filter based PGRP routing protocol for IoV network.

\section{References}

1. Acosta, A., and Espinosa, J. (2005) TraCI4Matlab: Enabling the Integration of the SUMO Road Traffic Simulator and Matlab Through a Software Re-engineering Process. In Modeling Mobility with Open Data, 155-170. DOI:10.1007\%2F978-3-319-15024-6_9.

2. Artimy, M., Robertson, W. and Phillips, W. (2005) Assignment of dynamic transmission range based on estimation of vehicle density. In: Proceedings of the 2nd ACM international workshop on Vehicular ad hoc networks, September 2005, Germany: ACM, 40-48.

3. Bao, X., Li, H., Zhao, G., Chang, L., Zhou, J. and Li, Y. (2020) Efficient clustering V2V routing based on PSO in VANETs. Measurement, 152, 1-21. Doi: 10.1016/j.measurement.2019.107306.

4. Boussoufa-Lahlah, S., Semchedine, F. and Bouallouche-Medjkoune, L. (2018) Geographic routing protocols for Vehicular Ad hoc NETworks (VANETs): A survey. Vehicular Communications, 11, 20-31. DOI: 10.1016/j.vehcom.2018.01.006.

5. Cheng, P., Lee, K., Gerla, M. and Härri, J. (2009) GeoDTN+Nav: Geographic DTN Routing with Navigator Prediction for Urban Vehicular Environments. Mobile Networks and Applications, 15(1), 61-82. DOI:10.1007/s11036-009-0181-6.

6. Karimi, R. and Shokrollahi, S. (2018) PGRP: Predictive geographic routing protocol for VANETs. Computer Networks, 141, 67-81. DOI: 10.1016/j.comnet.2018.05.017.

7. Karp, B. and Kung, H. (2000) GPSR: greedy perimeter stateless routing for wireless networks. In: MobiCom '00: Proceedings of the 6th annual international conference on Mobile computing and networking (MobiCom 2000), August 2000, Boston: IEEE, 243-245.

8. Lochert C., Hartenstein H., Tian J., Fussler H., Hermann D., and Mauve M. (2003) A routing strategy for vehicular ad hoc networks in city environments. In: Proceedings of IEEE IV2003 Intelligent Vehicles Symposium, July 2003, Columbus, OH, USA: IEEE.

9. Naumov, V. and Gross, T. (2007) Connectivity-Aware Routing (CAR) in Vehicular Ad-hoc Networks. In: Proceedings of 26th IEEE International Conference on Computer Communications, May 2007, Anchorage, AK, USA: IEEE, 1919-1927.

10. TOMTOM (2020) TomTom Traffic Index: Global Traffic Congestion Up as Bengaluru takes Crown of 'World's Most Traffic Congested City. Amsterdam, The Netherlands: TomTom. 